\title{
Commentary: Early Secondary Prevention of Cardioembolic Stroke with Direct Oral Anticoagulants (DOAC)
}

\author{
Kosmas Macha*, Bastian Volbers, Bernd Kallmünzer \\ Department of Neurology, Universitätsklinikum Erlangen, Friedrich-Alexander-University Erlangen-Nuremberg (FAU), Erlangen, Germany
}

Article Info

\section{Article Notes}

Received: January 09, 2017

Accepted: March 08, 2017

\section{*Correspondence:}

Kosmas Macha, MD

Department of Neurology, Universitätsklinikum Erlangen, Friedrich-Alexander-University Erlangen-Nuremberg (FAU), Schwabachanlage 6, 91054 Erlangen, Germany,

Email: kosmas.macha@uk-erlangen.de

(c) 2017 Macha K. This article is distributed under the terms of the Creative Commons Attribution 4.0 International License

\section{Keywords}

Oral anticoagulation

Ischemic stroke

Secondary prevention

Hemorrhagic transformation

DOAC

Atrial fibrillation

Review

\section{ABSTRACT}

Direct Oral Anticoagulants (DOAC) are highly effective for the prevention of systemic embolism and ischemic stroke in non-valvular atrial fibrillation, but the optimal time point of treatment initiation after stroke has not been defined yet. The very early period after stroke bears a particular high risk of stroke recurrence and the timely initiation of secondary prevention at that early stage could therefore be most beneficial for the patient. Contrarily the use of DOAC in the hyperacute phase of stroke might increase the risk of bleeding complications. The prospective trials investigating the use of DOAC versus vitamin $\mathrm{K}$ antagonists did not include patients during the first weeks after stroke due to safety concerns. However, preliminary results from smaller cohorts suggest that the use of DOACs in this early time frame is feasible and safe. Larger prospective trials are warranted to confirm these results and investigate the efficacy of early treatment initiation.

\section{Introduction}

Novel Direct Oral Anticoagulants (DOAC) are increasingly used for prevention of systemic embolism or ischemic stroke in patients with atrial fibrillation. In meta-analysis ${ }^{1}$ of the randomized trials (RE-LY, ROCKET AF, ARISTOTLE and ENGAGE AF-TIMI 48; n=42 411 DOACtreated patients) DOACs were associated with a significant reduction of stroke or systemic embolic events compared with vitamin K antagonists (VKA), mainly caused by a reduction of hemorrhagic stroke. DOACs also significantly reduced all-cause mortality and intracranial hemorrhage (ICH), but increased gastrointestinal bleeding. As the randomized trials of DOACs were mainly carried out in primary stroke-prevention, only a smaller proportion of patients had a history of previous transient ischemic attack (TIA) or ischemic stroke in these trials. Thus, there is only limited knowledge about secondary stroke prevention with DOACs, especially in the acute phase after an ischemic cerebrovascular event.

DOACs in Patients post TIA or Ischemic Stroke (Secondary Prevention)

Only $20.1 \%$ of DOAC-treated patients in RE-LY ${ }^{2}$ and $18.6 \%$ of DOACtreated patients in ARISTOTLE ${ }^{3,4}$ had a history of previous TIA or ischemic stroke. In ENGAGE AF-TIMI $48^{5} 28.3 \%$ of DOAC-treated participants had a history of a prior cerebrovascular ischemic event. Patients of ROCKET $\mathrm{AF}^{6,7}$ had in comparison to patients of the other randomized DOACtrials higher $\mathrm{CHADS}_{2}$-scores and $52.6 \%$ of DOAC-treated study patients had a history of previous TIA or ischemic stroke. However, results of subgroup-analysis of patients with prior TIA or ischemic stroke were similar to findings in the full cohort in all randomized trials $s^{8,7,4,9}$. To maximize safety in patients with prior ischemic stroke early therapy 
initiation post stroke was precluded by study design in all randomized trials. In ARISTOTLE ${ }^{3}$ a minimum of 7 days was necessary between an ischemic stroke and study inclusion. In both RE-LY ${ }^{2}$ and ROCKET $\mathrm{AF}^{6}$ patients during the first 14 days after an ischemic stroke were excluded. After severe, disabling stroke participation in RE-LY² was precluded for the first 180 days and in ROCKET $\mathrm{AF}^{6}$ for the first 90 days. In addition, TIA patients could not be included in ROCKET $\mathrm{AF}^{6}$ within 3 days of the event. The ENGAGE AF-TIMI 48 trial $^{5}$ even precluded inclusion of patients within 30 days of any ischemic stroke. Therefore, early secondary prevention was not investigated in the randomized DOAC-trials.

\section{Timing of Secondary Prevention - Safety and Efficacy}

Patients with atrial fibrillation and cerebral ischemia are at high risk for recurrent ischemic stroke especially in the early phase after stroke as the frequency of recurrent ischemic stroke related to atrial fibrillation could be demonstrated as high as $8 \%$ during the first 14 days ${ }^{10,11}$. Therefore it is desirable to initiate secondary prevention with oral anticoagulants as early as possible ${ }^{10}$. Based on their direct inhibition of thrombin (dabigatran) or factor Xa (rivaroxaban, apixaban, edoxaban) DOACs have a rapid onset of action (0.5-4 hours $)^{12}$. As patients in the acute phase after an ischemic stroke were precluded from the randomized trials by study design, there are no prospective data available regarding early secondary prevention of cardioembolic stroke with DOACs from a large patient cohort.

The SAMURAI-NVAF study ${ }^{13}$ demonstrated the safety of early DOAC-initiation in a Japanese-cohort of 466 patients as the investigators detected no ICH after DOAC-initiation within a median of four days post stroke.

Based on the results of their RAF study Paciaroni et $\mathrm{al}^{14}$ recommended an initiation of anticoagulation in the timeframe of day $4-14$ post stroke generally. Because of the poor prognosis of hemorrhagic complications in patients with posterior fossa ischemia they considered a delay of oral anticoagulation initiation beyond day 14 in these patients, similarly to patient with high risk of hemorrhagic transformation. In their study high $\mathrm{CHA}_{2} \mathrm{DS}_{2}$-VASc-score, high National Institutes of Health Stroke Scale (NIHSS)score, large ischemic lesion and type of anticoagulant (favoring oral anticoagulation alone without bridging) were predictive factors for primary study outcome (composite of stroke, transient ischemic attack, symptomatic systemic embolism, symptomatic cerebral bleeding and major extracranial bleeding). In 93 DOAC-treated study-patients therapy was initiated within a mean of $8.5 \pm 12.2$ days post index event and two (2.1\%) symptomatic ICHs and four $(4.3 \%)$ ischemic events (ischemic cerebrovascular event or systemic embolism) were detected within 90 days.

In their recent publication Seiffge et $\mathrm{al}^{15}$ detected no symptomatic ICH in all 155 patients receiving DOAC for secondary prevention of cardioembolic TIA or ischemic stroke, initiated median 5 days after the index event (65\% of patients received DOACs within 7 days). In the entire cohort (155 DOAC- and 49 VKA-treated patients) a total of six recurrent ischemic strokes occurred within 3 months; two in patients of the DOACearly group (DOAC initiation within 7 days; 5.1\%/year), two in the DOAClate group (DOAC initiation after 7 days; 9.3\%/year) and two in patients with VKA-therapy (11.3\%/year). One symptomatic ICH occurred in a VKA-treated patient. Thereby the rate of recurrent ischemic stroke after the index event $(n=6 ; 7.7 \% /$ year $)$ was 6 times higher than that of ICH $(n=1 ; 1.3 \% / y e a r)$. The authors could not show a significant difference in the rate of recurrent ischemic stroke between patients of the DOACearly and DOAClate groups (5.1\% vs. 9.3\%/year, $\mathrm{p}=0.53$ ).

We analyzed safety of our institutional, infarct sizebased treatment algorithm of early secondary prevention of cardioembolic stroke with DOACs (DOAC started immediately in TIA/minor stroke, within day 3-5 in $\leq 1 / 3$ middle cerebral artery (MCA)-territory, anterior cerebral artery (ACA)-territory, posterior cerebral artery (PCA)territory or infratentorial stroke, after $1-2$ weeks in $>1 / 3$ MCA-territory stroke) on the dataset of 243 consecutive cardioembolic TIA and ischemic stroke patients ${ }^{16}$. Here DOAC-therapy was initiated median on day 2 in TIA/minor stroke patients (group 1, $\mathrm{n}=41$; median age: 78.0 (73.5$84.5)$ years, female sex: $56.1 \%$, arterial hypertension $(\mathrm{AH})$ : 82.9\%, diabetes mellitus (DM): 22.0\%, median NIHSS-score: $0(0-1))$ and on day 4 and 5 in patients with non-extensive supratentorial (group 2 ( $\leq 1 / 3$ MCA-, ACA- or PCA-territory), $\mathrm{n}=170$; median age: $78.0(72.0-84.0)$ years, female sex: 45.3\%, AH: 90.0\%, DM: 33.5\%, median NIHSS-score: 5 (2-9), thrombolysis: $32.9 \%$ ) and infratentorial infarction (group 3, n=28; median age: 78.0 (67.8-80.0) years, female sex: 50.0\%, AH: 96.4\%, DM: 35.7\%, median NIHSS-score: 3 (1-5), thrombolysis: $17.9 \%$ ). In the small group of patients with extensive supratentorial infarction (group 4, >1/3 MCAterritory; $\mathrm{n}=4$; median age: $68.5(63.0-71.8)$ years, female sex: 75.0\%, AH: 75.0\%, DM: 0.0\%, median NIHSS-score: 15 (9-16), thrombolysis: 50.0\%) DOAC-therapy was initiated median after 1 week. Detecting two asymptomatic (0.8\%) and one symptomatic ICH $(0.4 \%)$, both in patients with non-extensive supratentorial infarction, no severe safety issues were observed in our in-patient cohort during the acute phase of DOAC-initiation (median length of hospital stay 5, 7, 9 and 13 days in groups 1-4, respectively). Hereby early initiation of DOAC-therapy was safe, even in the by hemorrhagic complications highly affected group of patients with fossa posterior infarction.

According to existing data and pathophysiological considerations a primary infarct size-based treatment algorithm (as shown above) or alternatively NIHSS-based treatment algorithm (as recommended by the European 
Society of Cardiology: TIA: 1 day after acute event; mild stroke (NIHSS <8): 3 days after acute event; moderate stroke (NIHSS 8-15): 6 days after acute event; severe stroke (NIHSS $\geq 16$ ): 12 days after acute event $)^{17}$ seems most reasonable for secondary prevention of cardioembolic stroke with DOACs. As strategically unfavorable located small infarctions can lead to a severe neurologic deficit an imaging-based treatment algorithm might be advantageous. However, the results of existing data are limited by the retrospective design with relevant selection bias and prospective trials are warranted.

In the future large registries (e.g. the GLORIA-AF Registry Program, NCT01468701), meta-analyses of existing data (e.g. Early start of direct, non-Vitamin $\mathrm{K}$ antagonist oral anticoagulants (DOAC) after recent ischemic stroke meta-analysis of individual patient data) and randomized trials (e.g. TIMING of Oral Anticoagulant Therapy in Acute Ischemic Stroke with Atrial Fibrillation, NCT02961348; Early versus Late initiation of direct oral Anticoagulants in post-ischaemic stroke patients with atrial fibrillation (ELAN)) will re-evaluate safety and efficacy of early secondary prevention of cardioembolic stroke with DOACs.

\section{Conclusion}

Data from smaller patient cohorts suggest feasibility and safety of early secondary prevention of cardioembolic stroke with DOACs. Larger randomized trials are warranted to confirm these results and investigate the efficacy of this approach for the prevention of early stroke recurrence.

\section{Acknowledgement}

We acknowledge support by Deutsche Forschungsgemeinschaft and Friedrich-Alexander-Universität Erlangen-Nürnberg (FAU) within the funding programme Open Access Publishing.

\section{Disclosures}

BV reported having received speaker honoraria and travel grants from Bayer and travel grants from BMS Pfizer and Boehringer Ingelheim. BK reported having received travel grants from Bayer, BMS Pfizer and Boehringer Ingelheim.

\section{References}

1. Ruff CT, Giugliano RP, Braunwald E, et al. Comparison of the efficacy and safety of new oral anticoagulants with warfarin in patients with atrial fibrillation: A meta-analysis of randomised trials. Lancet. 2014; 383: 955-962.

2. Connolly SJ, Ezekowitz MD, Yusuf S, et al. Dabigatran versus warfarin in patients with atrial fibrillation. N Engl J Med. 2009; 361: 1139-1151.
3. Granger CB, Alexander JH, McMurray JJV, et al. Apixaban versus warfarin in patients with atrial fibrillation. N Engl J Med. 2011; 365: 981-992.

4. Easton JD, Lopes RD, Bahit MC, et al. Apixaban compared with warfarin in patients with atrial fibrillation and previous stroke or transient ischaemic attack: A subgroup analysis of the aristotle trial. Lancet Neurol. 2012; 11: 503-511.

5. Giugliano RP, Ruff CT, Braunwald E, et al. Edoxaban versus warfarin in patients with atrial fibrillation. N Engl J Med. 2013; 369: 2093-2104.

6. Patel MR, Mahaffey KW, Garg J, et al. Rivaroxaban versus warfarin in nonvalvular atrial fibrillation. N Engl J Med. 2011; 365: 883-891.

7. Hankey GJ, Patel MR, Stevens SR, et al. Rivaroxaban compared with warfarin in patients with atrial fibrillation and previous stroke or transient ischaemic attack: A subgroup analysis of rocket af. Lancet Neurol. 2012; 11: 315-322.

8. Diener H-C, Connolly SJ, Ezekowitz MD, et al. Dabigatran compared with warfarin in patients with atrial fibrillation and previous transient ischaemic attack or stroke: A subgroup analysis of the re-ly trial. Lancet Neurol. 2010; 9: 1157-1163.

9. Rost NS, Giugliano RP, Ruff CT, et al. Outcomes with edoxaban versus warfarin in patients with previous cerebrovascular events. Findings From ENGAGE AF-TIMI 48 Effective Anticoagulation With Factor Xa Next Generation in Atrial Fibrillation-Thrombolysis in Myocardial Infarction 48. Stroke. 2016; 47: 2075-2082.

10. Kernan WN, Ovbiagele B, Black HR, et al. Guidelines for the prevention of stroke in patients with stroke and transient ischemic attack: A guideline for healthcare professionals from the american heart association/american stroke association. Stroke. 2014; 45: 2160 2236.

11. Berge E, Abdelnoor M, Nakstad PH, et al. Low molecular weight heparin versus aspirin in patients with acute ischaemic stroke and atrial fibrillation: A double-blind randomised study. Lancet. 2000; 355: 1205-1210.

12. Gómez-Outes A, Suárez-Gea ML, Lecumberri R, et al. Direct acting oral anticoagulants Pharmacology indications management and future perspectives. Eur J Haematol. 2015; 95: 389-404.

13. Toyoda K, Arihiro S, Todo K, et al. Trends in oral anticoagulant choice for acute stroke patients with nonvalvular atrial fibrillation in japan: The samurai-nvaf study. Int J Stroke. 2015; 10: 836-842.

14. Paciaroni M, Agnelli G, Falocci N, et al. Early recurrence and cerebral bleeding in patients with acute ischemic stroke and atrial fibrillation: Effect of anticoagulation and its timing: The raf study. Stroke. 2015; 46: 2175-82.

15. Seiffge DJ, Traenka C, Polymeris A, et al. Early start of doac after ischemic stroke Risk of intracranial hemorrhage and recurrent events. Neurology. 2016; 87: 1856-1862.

16. Macha K, Volbers B, Bobinger T, et al. Early initiation of anticoagulation with direct oral anticoagulants in patients after transient ischemic attack or ischemic stroke. J Stroke Cerebrovasc Dis. 2016; 25: 2317 2321.

17. Kirchhof P, Benussi S, Kotecha D, et al. 2016 esc guidelines for the management of atrial fibrillation developed in collaboration with eacts. Europace. 2016; 18: 1609-1678. 